\title{
Nucleon spectroscopy using multi-particle operators
}

\author{
Waseem Kamleh ${ }^{* \dagger}$ \\ Special Research Centre for the Subatomic Structure of Matter, \\ University of Adelaide, Australia \\ E-mail: waseem.kamleh@adelaide.edu.au
}

\section{Adrian Kiratidis}

Special Research Centre for the Subatomic Structure of Matter,

University of Adelaide, Australia

\section{Derek B. Leinweber}

Special Research Centre for the Subatomic Structure of Matter,

University of Adelaide, Australia

\begin{abstract}
The role of 5-quark operators in extracting the nucleon excited state spectrum via correlation matrix techniques is explored. In particular, the coupling of meson-baryon operators to nucleon resonance states and scattering states is studied. Results are presented for $2+1$ flavour dynamical ensembles in both the positive and negative parity channels. Fitting a single-state ansatz to the eigenstate-projected correlators provides robust energies for the low-lying spectrum that are essentially invariant across a variety of different operator bases. In particular, the resonant state energies obtained both with and without the use of meson-baryon operators agree, demonstrating that resonance energies can be reliably extracted solely using 3-quark operators.
\end{abstract}

The 32nd International Symposium on Lattice Field Theory

23-28 June, 2014

Columbia University New York, $N Y$

${ }^{*}$ Speaker.

$\dagger$ This research was undertaken with the assistance of resources at the NCI National Facility in Canberra, Australia, and the iVEC facilities at the University of Western Australia (iVEC@UWA), provided through the National Computational Merit Allocation Scheme and the University of Adelaide Partner Share. We also acknowledge the support of eResearch SA. This research is supported by the Australian Research Council. 


\section{Introduction}

The use of correlation matrix techniques $[1,2]$ to study the nucleon excited state spectrum has seen a significant amount of recent activity, with lattice studies being performed in both the positive parity $[3,4,5,6,7]$ and negative parity $[8,9,10]$ sectors. The underlying principle is to begin with a sufficiently large basis of $N$ operators (so as to span the space of the states of interest within the spectrum) and construct an $N \times N$ matrix of cross correlation functions,

$$
G_{i j}(t, \vec{p})=\sum_{\vec{x}} e^{-i \vec{p} \cdot \vec{x}}\left\langle\Omega\left|T\left\{\chi_{i}(x) \bar{\chi}_{j}(0)\right\}\right| \Omega\right\rangle .
$$

We then solve a generalised eigenproblem to find the linear combination of interpolating fields,

$$
\bar{\phi}^{\alpha}=\sum_{i=1}^{N} u_{i}^{\alpha} \bar{\chi}_{i}, \quad \phi^{\alpha}=\sum_{i=1}^{N} v_{i}^{\alpha} \chi_{i}
$$

such that $\phi^{\alpha}$ and $\bar{\phi}^{\alpha}$ couple to a single energy eigenstate (labelled by $\alpha$ ) and the correlation matrix is diagonalised,

$$
v_{i}^{\alpha} G_{i j}(t) u_{j}^{\beta}=\delta^{\alpha \beta} z^{\alpha} \bar{z}^{\beta} e^{-m_{\alpha} t} .
$$

The left and right vectors are then used to define the eigenstate-projected correlators (after applying the parity projection operators at $\vec{p}=0$ ),

$$
v_{i}^{\alpha} G_{i j}^{ \pm}(t) u_{j}^{\alpha} \equiv G_{ \pm}^{\alpha}(t) .
$$

At this point we note that if the operator basis does not appropriately span the low-lying spectrum $G_{ \pm}^{\alpha}(t)$ may contain a mixture of two or more energy eigenstates. There are a number of scenarios in which this might occur:

- At early Euclidean times the number of states strongly contributing to the correlation matrix may be (much) larger than the number of operators in the basis.

- There may be energy eigenstates present that do not couple or only couple weakly to the operators used. In particular, it is well known that local 3-quark interpolating fields couple poorly to multi-hadron scattering states.

- The nature of the operators selected may be such that it is not possible to construct a linear combination with the appropriate structure to isolate a particular state.

It is important to have a strategy to ensure that one can accurately obtain eigenstate energies from the correlation matrix. Now, even if a mixture of states is present, asymptotically Euclidean time evolution will tend to isolate a single state,

$$
G_{ \pm}^{\alpha}(t) \stackrel{t \rightarrow \infty}{=} z_{\alpha} \bar{z}_{\alpha} e^{-m_{\alpha} t}
$$

Hence, the method we use is to construct effective masses of different states from the eigenstateprojected correlators and then analyse them in the usual way. As we will demonstrate, a careful $\chi^{2}$ analysis to fit the single-state ansatz ensures a robust extraction of the eigenstate energies. 

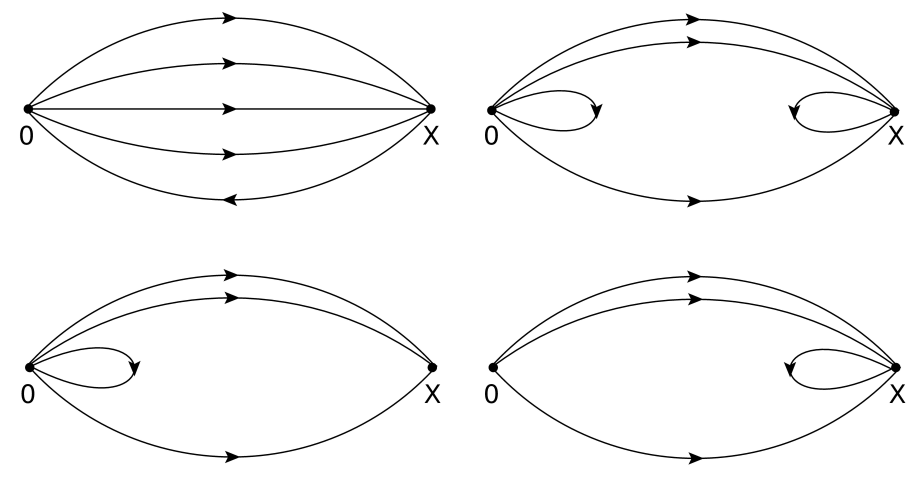

Figure 1: The Feynmann diagrams present following the introduction of five-quark interpolating fields.

As described above, the choice of an appropriate operator basis is critical to obtaining the complete spectrum of low-lying excited states. Recall that we can expand any radial function using a basis of Gaussians of different widths $f(|\vec{r}|)=\sum_{i} c_{i} e^{-\varepsilon_{i} r^{2}}$. This leads to the use of Gaussiansmeared fermion sources with a variety of widths [11], providing an operator basis that is highly suited to accessing radial excitations. Indeed, the CSSM lattice collaboration has used this well established technique $[6,9]$ in the calculation of the positive and negative parity nucleon excited state spectra. It is the combination of Gaussian sources of different widths that allows for the formation of the nodal structures needed to isolate the different radial excitations [12].

However, it has been shown that a basis consisting solely of three-quark operators has difficulty in detecting multi-particle scattering energy levels [10]. The coupling of three-quark baryon operators to multi-particle states is strongly suppressed, leading us to consider the inclusion of interpolating fields that we expect to have substantial overlap with multi-particle meson-baryon type states [8]. One possible solution is to explicitly include hadron-hadron type interpolators, as has been done with mesons [13] by combining single-hadron operators with the relevant momentum. This creates an operator that necessarily has a high overlap with the scattering state of interest, enabling its extraction.

In this work we take a different approach, and instead aim to construct meson-baryon type interpolators without explicitly projecting momenta to investigate the role that the resulting operator plays in the calculation of the nucleon spectrum. Using these operators we perform spectroscopic calculations using a variety of bases containing both three- and five-quark operators. Examining the resulting spectra provides us with an excellent opportunity to test the robustness of the variational techniques employed.

\section{Simulation Details}

For this work we use the PACS-CS $2+1$ flavour dynamical-fermion configurations [14] made available through the ILDG [15]. These configurations use the non-perturbatively $\mathscr{O}(a)$-improved Wilson fermion action and the Iwasaki gauge action. The lattice size is $32^{3} \times 64$ with a lattice spacing of $0.0907 \mathrm{fm}$ providing a physical volume of $\approx(2.90 \mathrm{fm})^{3} . \beta=1.90$, the light quark mass is set by the hopping parameter $\kappa_{u d}=0.13770$ which gives a pion mass of $m_{\pi}=293 \mathrm{MeV}$, while the strange quark mass is set by $\kappa_{s}=0.13640$. Gauge-invariant Gaussian smearing [16] is 
employed at the source and sink which alters the overlap of the operator with the various states in the spectrum. The source is inserted at $t_{s}=16$, while the variational analysis is done at $t_{0}=$ 17 and $d t=3$ which provides a good balance between systematic and statistical uncertainties. Uncertainties are obtained via single elimination jackknife while a full covariance matrix analysis provides $\chi^{2} /$ dof which is utilized to select fits.

We make use of the following conventional three-quark nucleon operators,

$$
\begin{aligned}
& \chi_{1}=\varepsilon^{a b c}\left[u^{a T}\left(C \gamma_{5}\right) d^{b}\right] u^{c}, \\
& \chi_{2}=\varepsilon^{a b c}\left[u^{a T}(C) d^{b}\right] \gamma_{5} u^{c} .
\end{aligned}
$$

Utilising the Clebsch-Gordon coefficients to project to $I=1 / 2, I_{3}=+1 / 2$, we can write down the general form of our meson-baryon interpolating fields [17],

$$
\begin{aligned}
\chi_{N \pi}(x)=\frac{1}{2 \sqrt{3}} \varepsilon^{a b c}\{ & 2\left(u^{T a}(x) \Gamma_{1} d^{b}(x)\right) \Gamma_{2} d^{c}(x)\left[\bar{d}^{e}(x) \gamma_{5} u^{e}(x)\right] \\
& -\left(u^{T a}(x) \Gamma_{1} d^{b}(x)\right) \Gamma_{2} u^{c}(x)\left[\bar{d}^{e}(x) \gamma_{5} d^{e}(x)\right] \\
+ & \left.\left(u^{T a}(x) \Gamma_{1} d^{b}(x)\right) \Gamma_{2} u^{c}(x)\left[\bar{u}(x)^{e} \gamma_{5} u^{e}(x)\right]\right\},
\end{aligned}
$$

providing us with two five-quark operators, denoted $\chi_{5}$ and $\chi_{5}^{\prime}$ which correspond to $\left(\Gamma_{1}, \Gamma_{2}\right)=$ $\left(C \gamma_{5}, \mathrm{I}\right)$ and $\left(\Gamma_{1}, \Gamma_{2}\right)=\left(C, \gamma_{5}\right)$ respectively. Note, these transform negatively under parity, so we multiply our five-quark operators by $\gamma_{5}$ before constructing the correlation matrix.

We form seven operator bases by selecting different combinations of the four nucleon interpolating fields as outlined in the following table:

\begin{tabular}{cc}
\hline Basis Number & Operators Used \\
\hline 1 & $\chi_{1}, \chi_{2}$ \\
2 & $\chi_{1}, \chi_{2}, \chi_{5}$ \\
3 & $\chi_{1}, \chi_{2}, \chi_{5}^{\prime}$ \\
4 & $\chi_{1}, \chi_{2}, \chi_{5}, \chi_{5}^{\prime}$ \\
5 & $\chi_{1}, \chi_{5}, \chi_{5}^{\prime}$ \\
6 & $\chi_{2}, \chi_{5}, \chi_{5}^{\prime}$ \\
7 & $\chi_{5}, \chi_{5}^{\prime}$ \\
\hline
\end{tabular}

The presence of creation quark fields in our annihilation interpolating field and vice-versa leads to the requirement of calculating the loop propagators, present in the right-hand diagram of Figure 1. There are a number of ways of dealing with such diagrams including distillation [18], and the Laplacian Heaviside (LapH) smearing method [19]. Here we will stochastically estimate inverse matrix elements fully diluting in spin, colour and time.

Throughout this work we employ two different levels of fermion source and sink smearing to increase the basis size and provide access to radial excitations. We use $n_{s}=35$ and $n_{s}=200$ sweeps of smearing providing bases of sizes 4,6 and 8 . 


\section{Results}

We now present the nucleon spectrum in the positive parity sector in Figure 2. The solid horizontal lines therein have been added to guide the eye, having their values set by the states in basis number 4 , since this basis contains all the operators studied and hence possess the largest span.

Of particular interest is the robustness of the variational techniques employed. While changing bases may effect whether or not a particular state is seen, the energy of the extracted states is consistent across the different bases, even though they contain qualitatively different operators.

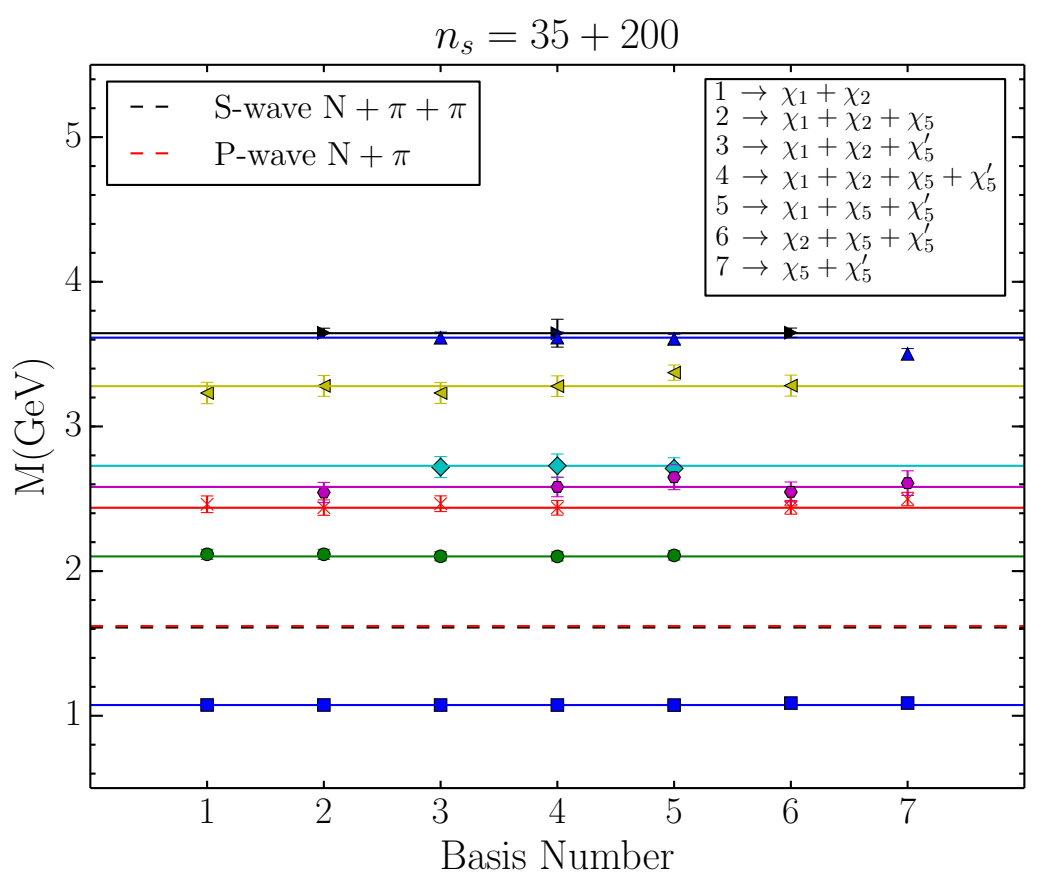

Figure 2: Positive parity nucleon spectrum with various operator bases with 35 and 200 sweeps of smearing. Horizontal solid lines are present to guide the eye and are drawn from the central value of the states in basis 4 , since this basis is the largest.

The ground-state nucleon is observed in every basis regardless of the absence or presence of a particular operator. In contrast, we see that $\chi_{1}$ is critical to the extraction of the first excited state, a radial excitation of the ground state [12]. We see this state in bases 1 through 5, whereas bases 6 and 7 which lack $\chi_{1}$ do not observe this state.

Despite the use of 5-quark operators, states consistent with the P-wave $N \pi$ or S-wave $N \pi \pi$ scattering thresholds are absent. This is understood by noting that none of our operators have a source of the back-to-back relative momentum necessary to observe the scattering states in this channel.

The negative parity nucleon spectrum is presented in Figure 3. Again solid horizontal lines have been added to guide the eye, having their values set by the states in basis number 4 . Once again, while changing bases effects whether or not we observe a given state, the extracted states display an impressive level of consistency. In accord with previous studies [9, 20], we find that the 
$\chi_{1}$ interpolating field is crucial for extracting the lowest negative parity resonance, associated with the $S_{11}(1535)$, as we do not observe this state when $\chi_{1}$ is absent as in bases 6 and 7.

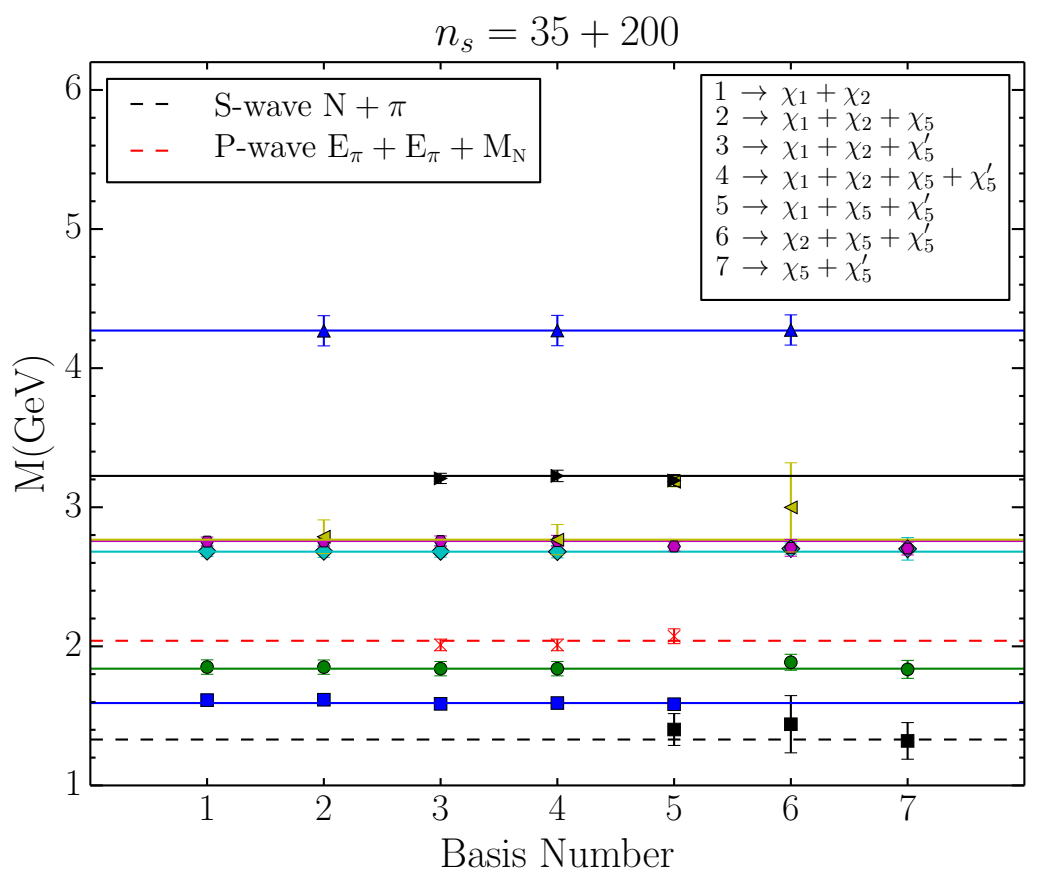

Figure 3: The negative parity nucleon spectrum with various operator bases using 35 and 200 sweeps of smearing. Solid horizontal lines are present to guide the eye and are drawn from the central value of the states in basis 4 , since this basis is the largest. The variational parameters used herein are $\left(t_{0}, d t\right)=(17,3)$.

In contrast to the positive parity results, we do observe states that are consistent with the scattering thresholds in the negative parity channel, specifically the S-wave $N \pi$ (bases $5,6,7$ ) and P-wave $N \pi \pi$ (bases 3,4,5) thresholds. It is somewhat surprising that basis 4 fails to see the $\mathrm{S}$-wave $N \pi$ scattering threshold, despite being the largest. It seems that the coupling to this scattering state is low relative to the large spectral strength of the resonant states. We observe that a scattering state is seen if (and only if) the $\chi_{5}^{\prime}$ operator is included, indicating that the presence of the vector di-quark in the interpolator may play a significant role in scattering state excitation.

It is important to note that even after the introduction of operators that permit access to lowlying scattering states in the spectrum (such as in bases 5, 6 and 7) the energies of the higher states in the spectrum are consistent, demonstrating the robustness of the variational techniques employed.

\section{Conclusion}

We studied the excited state nucleon spectrum on a variety of bases including both standard 3-quark operators and local multi-particle operators. We find that fitting a single-state ansatz to the effective masses of the eigenstate projected correlators results in a robust extraction of the spectrum in both the positive and negative parity channels. While the selection of states that were observed 
varied between bases, when a given state was seen the extracted energy agreed across qualitatively different bases.

An important feature of our negative parity results is that the energies of the resonance states extracted are consistent across all bases in which the state is observed, regardless of the presence (or not) of a lower-lying scattering threshold state in the correlation matrix analysis. This study demonstrates that (by using the techniques described) one does not need to have access to the scattering states to reliably extract resonant state energies.

\section{References}

[1] C. Michael Nucl.Phys. B259 (1985) 58.

[2] M. Luscher and U. Wolff Nucl.Phys. B339 (1990) 222-252.

[3] D. S. Roberts, W. Kamleh, and D. B. Leinweber Physics Letters B 725 (2013), no. 1-3 164-169, [1304.0325].

[4] R. G. Edwards, J. J. Dudek, D. G. Richards, and S. J. Wallace Phys.Rev. D84 (2011) 074508, [1104.5152].

[5] M. Mahbub, A. O. Cais, W. Kamleh, B. G. Lasscock, D. B. Leinweber, et al. Phys.Lett. B679 (2009) 418-422, [0906.5433].

[6] M. S. Mahbub, W. Kamleh, D. B. Leinweber, P. J. Moran, and A. G. Williams Phys.Lett. B707 (2012) 389-393, [1011.5724].

[7] K.-F. Liu, Y. Chen, M. Gong, R. Sufian, M. Sun, et al. PoS LATTICE2013 (2014) 507, [1403.6847].

[8] C. Lang and V. Verduci Phys.Rev. D87 (2013), no. 5 054502, [1212. 5055$].$

[9] M. S. Mahbub, W. Kamleh, D. B. Leinweber, P. J. Moran, and A. G. Williams Phys.Rev. D87 (2013) 011501, [1209.0240].

[10] M. S. Mahbub, W. Kamleh, D. B. Leinweber, and A. G. Williams Annals Phys. 342 (2014) 270-282, [1310.6803].

[11] T. Burch et al. Phys.Rev. D70 (2004) 054502, [hep-lat/ 040500 6].

[12] D. S. Roberts, W. Kamleh, and D. B. Leinweber Phys.Rev. D89 (2014) 074501, [1311. 6626].

[13] C. Morningstar, J. Bulava, B. Fahy, J. Foley, Y. Jhang, et al. Phys.Rev. D88 (2013), no. 1 014511, [1303.6816].

[14] PACS-CS Collaboration, S. Aoki et al. Phys.Rev. D79 (2009) 034503, [0807.1661].

[15] M. G. Beckett, B. Joo, C. M. Maynard, D. Pleiter, O. Tatebe, et al. Comput.Phys.Commun. 182 (2011) 1208-1214, [0 910 .1692].

[16] S. Gusken Nucl.Phys.Proc.Suppl. 17 (1990) 361-364.

[17] A. L. Kiratidis, W. Kamleh, and D. B. Leinweber PoS LATTICE2012 (2012) 250, [1301. 3591 ].

[18] Hadron Spectrum Collaboration, M. Peardon et al. Phys.Rev. D80 (2009) 054506, [0 905 . 2160].

[19] C. Morningstar, J. Bulava, J. Foley, K. J. Juge, D. Lenkner, et al. Phys.Rev. D83 (2011) 114505, [1104.3870].

[20] M. S. Mahbub, W. Kamleh, D. B. Leinweber, P. J. Moran, and A. G. Williams Phys.Rev. D87 (2013), no. 9 094506, [1302.2987]. 